\title{
BIOG-BYWORD
}

\section{Study on Allergic Rhinitis of Artemisia Sphaerocephala}

\author{
Jingmin Niu1, 2, Xuan Gong1, Lizhi Niu'* \\ ${ }^{1}$ Beijing University of Chinese Medicine, Beijing 100029, China \\ ${ }^{2}$ Chifeng Municipal Hospital, Chifeng 024000, Inner Mongolia, China \\ *Corresponding author: Lizhi Niu, 18811132508@163.com
}

\begin{abstract}
Objective: To explore the cause and feasibility of Artemisia ordosica allergic rhinitis. Methods: The main allergic causes of allergic rhinitis patients in Chifeng City of inner Mongolia were analyzed by a control method. Results: According to the results, Artemisia ordosica is the most important cause of allergic rhinitis in inner Mongolia. In addition, allergic history, asthma and other diseases, or animal hair and other factors can also increase the risk of patients with allergic rhinitis. Conclusions: Artemisia sphaerocephala is the main cause of allergic rhinitis. In recent years, allergic rhinitis has attracted the attention of international organizations, and the medical community has also studied more methods for the treatment of rhinitis, such as putting forward relevant traditional Chinese medicine (TCM) treatment methods.
\end{abstract}

Keywords: Artemisia ordosica L; Rhinallergosis; Allergens; Sensitization; Related research

Publication date: July 2021; Online publication: July 31, 2021

\section{Introduction}

With the acceleration of urbanization, living standards and medical conditions have been improved. Since, the air around the city is constantly polluted, there is an increasing number of people whom are allergic to rhinitis and possibly already allergic to rhinitis. Allergic rhinitis caused by cephalosporins has become a hot topic of public concern. Research shows that in the past two years, the outbreak season of allergic rhinitis mainly concentrated in July to October, and the number of rhinitis increased sharply. Most of the root causes of allergic rhinitis are local wild plants. The fact shows that cephalosporin is the real allergen in allergic rhinitis. Allergic rhinitis is an immune disease caused by exogenous antigens. Hyperallergens first produce immunoglobulin $\mathrm{E}$ in the body through contact with allergic patients, thus making the body sensitive ${ }^{[1]}$. Nasal congestion, itching and persistent sneezing were the most common clinical symptoms.

Secondly, patients will have obvious nasal hydration and olfactory dysfunction, which will eventually affect the sleep of patients and increase the family economy of patients. Due to its complex symptoms and intractable rhinitis, it has become an important research and disease prevention target of international organizations in the 21 st century. The incidence rate of rhinitis is increasing year by year. The key challenge in treatment is to explore the cause of rhinitis. There are differences in climate, geographical environment and plants, and the common allergens leading to allergic rhinitis are also very different. This study selected 200 cases of patients with allergic rhinitis in our hospital (Chifeng Municipal Hospital), through retrospective case data analysis and allergen sensitization related factors in patients with allergic rhinitis, and suggested the relevant Chinese herbal medicine treatment, now the report is as follows. 


\section{Materials and methods}

\subsection{Source of cases}

Chifeng City, Inner Mongolia Autonomous Region was selected as the research area. From 2019 to 2020, 200 patients with allergic rhinitis were treated in our hospital, whom were 100 males and 100 females, aged from 6 to 60 years old. The inclusion criteria were that all patients had typical paroxysmal sneezing, runny nose, stuffy nose and other symptoms within at least 2 weeks. During the treatment, all patients were randomly and spontaneously tested, and their families were informed of the relevant situation and signed the agreement.

\subsection{Test method}

The allergen detection system was used in the system. The components of the system were as follows: High speed reader, incubator and fixed frequency mixer. With the advantages of repeatability and high sensitivity, the detection system brings high efficiency for detection. In the process of test reagent selection, the test reagent strip was mixed group of allergen screening combination. Artemisia, dust, pig grass, animal hair, hay powder, dandelion, pepper, seafood, Aspergillus fumigatus, etc., were some of the allergens used.

\subsection{Inspection principle}

The allergen was adsorbed on the surface of the nitrocellulose membrane and placed in the reaction tank. After the patient serum was added into the pipette and incubated at room temperature, the allergen specific $\mathrm{IgE}$ antibody in the sample reacted with the allergen and bounded to the nitrocellulose membrane. After eluting the excess antibody, biotin labeled anti human $\operatorname{IgE}$ antibody was added and incubated at room temperature to elute the unbound antibody. Then, the cells were incubated with nisin labeled alkaline phosphatase at room temperature and combined with nisin and biotin. Then washed the unbound enzyme labeled antibiotic streptomycin ${ }^{[2]}$. After BCIP / NBT substrate was added and incubated, a specific enzyme color reaction occurred, and precipitation appeared on the reagent strip, and the color concentration was proportional to the content of $\operatorname{IgE}$ antibody in serum. When the reagent strip was dry, it was checked with a special allergen detector, and the quantitative test results were read.

\subsection{Statistical methods}

The sample rate of each group was analyzed as $\mathrm{X}^{2}$ test. When $\mathrm{P}<0.01$, the difference was statistically significant.

\section{Results}

\subsection{Test results of related allergens}

The top five allergens were: Artemisia sphaerocephala (55.78\%), dust (22.85\%), ragweed (21.26\%), animal hair $(16.93 \%)$ and hay dust $(12.7 \%)$. The test results showed that the serum total IgE values of patients with allergic rhinitis were positive, as shown in Table 1.

At the same time, the detection period was from 2019 and 2020. In July, August, September and October of the two years, the allergen detection cases show an upward trend, and then begin to slowly decline in October (as shown in Figure 1.). 
Table 1. Distribution of allergens in allergic rhinitis

\begin{tabular}{cc}
\hline Allergen & Positive rate [n (\%)] \\
\hline Artemisia sphaerocephala & $156(55.78)$ \\
Dust & $130(22.85)$ \\
Ragweed & $109(21.26)$ \\
Animal hair & $93(16.93)$ \\
Hay dust & $79(12.7)$ \\
Dandelion & $55(11.66)$ \\
Pepper & $33(9.33)$ \\
Seafood & $24(6.89)$ \\
Aspergillus fumigatus & $10(5.78)$ \\
\hline
\end{tabular}

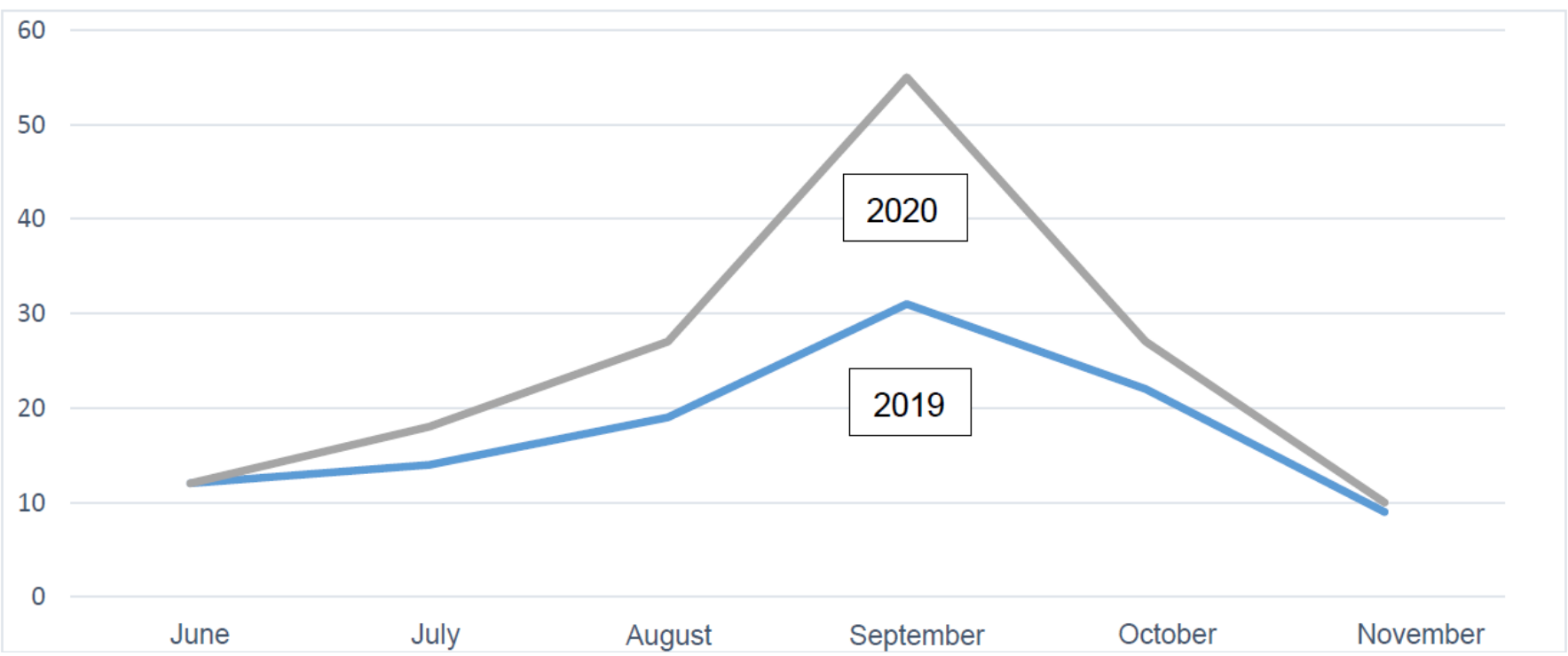

Figure 1. Distribution of the number of patients with allergic rhinitis in Chifeng City (June 2019 to November 2020)

\section{Discussion}

In recent years, with the acceleration of urbanization, negative effects have been increasing. For example, incidence rate of allergic rhinitis city has increased year by year. Allergic rhinitis seriously affects people's normal life, thus has been paid more attention by the international health organization. At present, the cause of allergic rhinitis has not been studied. There are different views on allergic rhinitis. Medical researchers believe that genetic and environmental factors are important causes of allergic rhinitis. In this paper, a casecontrol study was used to analyze the sensitization factors of patients with allergic rhinitis ${ }^{[3]}$. There are two kinds of allergen detection methods, namely "in vivo" measurement and "in vitro" measurement. Both methods can detect allergen specific IgE for data analysis. In vitro has the advantages of high detection efficiency, reliable parameters, not affected by drugs, no systemic reaction risk, and is gradually being popularized and applied in clinical. The selected area was Chifeng City, Inner Mongolia Autonomous 
Region, from 2019 to 2020 . A total of 200 patients with allergic rhinitis were admitted, including 100 males and 100 females. The age is 6-60 years old. The inclusion criteria were all patients with typical paroxysmal sneezing, runny nose, nasal congestion and other symptoms within at least 2 weeks. During the treatment, all patients underwent random and voluntary examination, and their families understood the relevant situation and signed the contract.

\subsection{The study results}

The total IgE in the serum of patients with allergic rhinitis was $60.78 \%$, and it should change at least twice at the same time. Due to the diversity of allergens, there were many unknown allergens, and the types of allergens used in detection reagents were limited. The results of allergen test showed that the total serum IgE of 200 patients with allergic rhinitis was positive. The top five allergens were cephalosporin, with a positive rate of $55.78 \%$, dust, pig grass, animal hair and hay powder of $22.85 \%, 21.26 \%, 16.93 \%$ and $12.7 \%$, respectively. Studies and tests show that Artemisia argyi has the highest content and higher concentration. Meanwhile, the selected measurement periods were 2019 and 2020. In the past two years, the number of allergen testing cases increased in July, August, September and October, and then decreased gradually in October. In September, it indirectly reached the peak in Chifeng City, indicating that the number of patients with allergic rhinitis increased in September.

Cephalosporin is the main cause of allergic rhinitis. The incidence of allergic rhinitis and the types of allergens may vary from region to region due to the geography, climate and particularity of plants. The results of this study show that Artemisia argyi plants were highly correlated with seasonal allergic rhinitis in summer and autumn, which provides a basis for environmental protection and optimization of vegetation structure in this area ${ }^{[2]}$. The epidemiological survey of allergic rhinitis showed that compared with other regions at home and abroad, the overall annual prevalence rate of allergic rhinitis in that region was lower and did not increase significantly. July, August, September and October were the most prevalent periods, so cases of allergic rhinitis were increasing. With the continuous development of the health care industry, allergic rhinitis has been alleviated. Recently, allergic rhinitis has attracted the attention of international organizations, and the medical community was also studying more treatment methods for rhinitis, such as recommending related herbal treatment methods. The symptoms and signs of allergic rhinitis treated by acupuncture were various, including systemic acupuncture, electric acupuncture, moxibustion and etc. In addition, the clinical effect of non-acupuncture and acupuncture was more important. It can also be used in the treatment of allergic rhinitis. Chinese herbal medicine comprehensive treatment is the comprehensive treatment of allergic rhinitis. In addition to reducing the symptoms and signs of rhinitis, it can also significantly improve the physical sensitivity of patients. In short, Artemisia argyi is an important cause of allergic rhinitis, combined with herbal medicine it can effectively improve the symptoms of rhinitis.

\section{Disclosure statement}

The author declares no conflict of interest.

\section{References}

[1] Li P, 2017, Northern Area Allergic Rhinitis High Incidence, is Artemisia Really the Culprit?. Beijing Science and Technology Daily, (42).

[2] Peng YP, Kang QQ, Liu LZ, 2015, Study on Related Factors of Allergen Sensitization in Children with Allergic Rhinitis. Journal of Yan'an University (Medical Science Edition), 13(2): 27-29.

[3] Xu Y, 2017, Study on the Effect of Volatile Oil from Artemisia Rupestris on Allergic Rhinitis, Xinjiang Medical University, Xinjiang, DOI:10.7666/d.D01196096. 